\title{
Evolution of Pair Correlation Symmetries and Supercurrent Reversal in Tilted Weyl Semimetals
}

\author{
Mohammad Alidoust ${ }^{1}$ and Klaus Halterman ${ }^{2}$ \\ ${ }^{1}$ Department of Physics, K.N. Toosi University of Technology, Tehran 15875-4416, Iran \\ ${ }^{2}$ Michelson Lab, Physics Division, Naval Air Warfare Center, China Lake, California 93555, USA
}

(Dated: January 15, 2020)

\begin{abstract}
We study the effective symmetry profiles of superconducting pair correlations and the flow of charge supercurrent in ballistic Weyl semimetal systems with a tilted dispersion relation. Utilizing a microscopic method in the ballistic regime and starting from both opposite-pseudospin (the band degree of freedom) and equalpseudospin phonon-mediated opposite-spin electron-electron couplings, we calculate the anomalous Green's function to study various superconducting pair correlations that Weyl semimetal systems may develop. The momentum-space profile reveals that by properly manipulating the parameters of Weyl semimetal systems, including the tilting parameter, the effective symmetry class of even-parity $s$-wave (odd-parity $p$-wave) superconducting correlations can be converted into a $d$-wave ( $f$-wave) symmetry class that consists of equal-pseudospin and opposite-pseudospin channels. We also find that the supercurrent in a ballistic Weyl Josephson junction can be made to vanish or switch directions, depending on the tilt of the Weyl cones, in addition to the relevant parameters characterizing the Weyl semimetal and junction. We show that inversion symmetry breaking terms introduce transitions that result in the appearance of self-biased current at zero difference between the macroscopic phases of the superconducting segments, creating a $\varphi_{0}$ Josephson state. Weyl semimetal systems are shown to offer several experimentally tunable parameters to control the induction of higher harmonics into the current phase relations.
\end{abstract}

\section{INTRODUCTION}

A Weyl semimetal (WS) is a topologically nontrivial phase of matter that has been heavily pursued experimentally and theoretically. ${ }^{1-3}$ The band structure in these materials exhibits Weyl points, where the conduction and valence bands intersect. Around these points, the energy dispersion of the quasiparticle excitations has a conical profile. The Weyl points only appear in pairs, which are identified by Weyl fermions in real space, or a source or sink of Berry curvature in momentum space. ${ }^{4,5}$ This topological phase results in the band structure in the bulk of Weyl semimetals being gapless, although Fermi arc states connect pairs of Weyl points. ${ }^{6}$ As has been widely discussed, the presence of gapless surface states opens up transport channels free from backscattering. For a conventional type-I WS, the Fermi surface is point-like, where electron and hole states occupy separate energy ranges above or below the Weyl point. ${ }^{7}$ By tilting the Weyl cones through the tilt parameter $\beta$, a type-II WS emerges with an open Fermi surface containing electron and hole pockets near the Weyl points. ${ }^{8}$ Recently, some experimental signatures of the type-II WS phase have been reported. ${ }^{9}$ Also, the optical properties of type-II WSs have been investigated ${ }^{10-14}$, including how tilt affects optical conductivity ${ }^{10}$, absorption ${ }^{11,13}$, and Hall conductivity. ${ }^{12}$

If the Weyl semimetal is intrinsically superconducting, or becomes so through proximity effects, it can host surfacelocalized Majorana fermions with zero energy. ${ }^{15,27,29}$ Since Majorana fermions follow non-Abelian statistics and are their own antiparticles, superconducting Weyl systems are anticipated to play a promising role in topological quantum computing. ${ }^{16-19}$ Experimentally, superconductivity in Weyl materials has been discovered in a variety of settings, including in $\mathrm{MoTe}_{2}$ under pressure, and in TaAs by photoemission spectroscopy. ${ }^{20,21}$ It was reported that surface superconductivity can be induced in NbAs through ion irradiation. ${ }^{22} \mathrm{Re}-$ cently, $\mathrm{Nb}$-doped $\mathrm{Bi}_{2} \mathrm{Se}_{3}$ and the heavy fermion superconductor $\mathrm{UPt}_{3}$ were predicted to be Weyl superconductors. ${ }^{23}$ Apart from naturally occurring materials, superconductivity in Weyl semimetals may also be realized through doping ${ }^{24-26}$. These intriguing phenomena have motivated a significant wave of interest in Weyl metal systems both theoretically and experimentally. ${ }^{20,27-31,34-66}$

When discussing the superconducting state of a Weyl semimetal, the symmetry of the electron-electron coupling potential, $\Delta$, is an important property that gives insight into the nature of the pairing correlations. Moreover, when a relationship exists between the quasiparticle excitations in the Weyl phase and the pair correlations, a more complete understanding of the underlying electronic structure can be obtained. As a typical example, hybrid superconducting systems containing inhomogenous ferromagnets can generate odd-frequency equal-spin triplet pairs. In these systems, the triplet pairs are accompanied by a zero energy peak in the density of states, which might be a signature of spin-triplet superconducting correlations. For an overview of this phenomenon, see Refs. 67-72 and references therein. The dominant pairing symmetry in a system however, depends on many factors, including the band structure and strength of the pairing interactions. Thus, by implementing complimentary experimental probes that identify thermodynamic or spectroscopic signatures, the pairing symmetries and associated pairing mechanisms and types of interactions can be clarified.

A macroscopic quantum effect that can reveal experimental signatures of the electronic properties of the Weyl semimetal is the Josephson current, which arises from a phase difference across two superconducting segments in a junction. Applications involving Josephson junctions have grown due to their impact and potential improvements in next generation superconducting computing, nonvolatile memories, and magnetometers ${ }^{73}$, where single flux quantum circuits contain numerous arrangements of Josephson junctions to improve 
speed and sensitivity. To determine the thermodynamic properties of practical cryogenic Weyl devices, it is crucial to understand the behavior of charge current flow in these types of systems.

This paper contains two main parts. Since the superconducting pair correlations can reveal hints of the host material, including lattice interactions, electronic structure, and pairing mechanisms, the symmetry of the superconducting correlations can play a significant role in most experimental tests. Thus, first we study the symmetry profiles of the superconducting correlations that a tilted WS (in the type-I and type-II phases) might develop through opposite-pseudospin (Pspin the band degree of freedom) and equal-pseudospin phononmediated opposite-spin electron-electron coupling. To study the symmetries of the superconducting correlations, we construct the Green's function in the ballistic regime. We derive analytical expressions for the Green's function components and explore both analytically and numerically the behavior of the pair correlations for a variety of WS system parameters. Our results show that, depending on the parameters, a WS can support superconducting pair correlations with effective $s$-wave and $p$-wave symmetry classes that are evenparity and odd-parity, consisting of opposite-Pspin and equalPspin channels. Importantly, we find that by properly tuning the parameters of a WS, including the tilting parameter $\beta$, the effective $s$-wave and $p$-wave symmetry classes can evolve into $d$-wave and $f$-wave classes. Thus, our results suggest that this type of superconducting WS platform can serve as a "switch" that converts the symmetry classes of the superconducting correlations. These predictions can be confirmed by high resolution angular point-contact spectroscopy experiments or Meissner-effect based experiments that are suitable to probe the directional-dependency of superconducting correlations. The findings here may also stimulate further studies involving scenarios similar to a recent experiment where superconductivity in a Weyl semimetal subject to external strain was studied ${ }^{66}$.

The second part of this paper is devoted to the transport of dissipationless charge current. We make use of wavefunctions derived from an exact diagonalization of the Bogoliubov-de Gennes Hamiltonian, without applying any simplifying approximations. A phase difference $\varphi$ is established across a Josephson junction to study the behavior of the supercurrent in a superconductor $(\mathrm{S})$-normal metal $(\mathrm{N})$ - superconductor $(\mathrm{S})$ junction. We consider the situation where superconductivity arises from unequal spin quasiparticles belonging to different Pspins. We find that by varying various parameters, including $\beta$, and the inversion breaking parameters of the WS, the system can experience a transition whereby the current reverses direction and changes its periodicity from $2 \pi$ to $4 \pi$ at the reversal points. The inclusion of a proper inversion breaking parameter creates a situation where a self-biased current can flow through the junction with $\varphi=0$, creating a $\varphi_{0}$ Josephson state. Our results demonstrate that the self-biased supercurrent is controllable by different parameters of the WS junction, including $\beta$ and junction length. In contrast to previous works where an exchange field is a necessary ingredient ${ }^{29,30,77-79}$, the $\varphi_{0}$ state explored here relies on inherent parameters of the WS Josephson junction independent of the density of nonmagnetic impurities and disorder that might be introduced in the system ${ }^{28}$. Our results in the ballistic regime are consistent with those explored in the diffusive limit of the quasiclassical regime $^{28}$.

In Sec. II, we outline the model Hamiltonian used and we establish the Green's function formalism for calculating the pairing correlations that can inhabit a Weyl semimetal with the possibility of a tilting parameter that can render the WS to a type-II phase. We also discuss the opposite-Pspin and equalPspin phonon-mediated electron-electron coupling model that is used to derive the components of the Green's function. We next utilize a quantum mechanical approach to find the supercurrent in a Josephson junction structure comprised of a WS. In Sec. III, we present results for both the pairing correlation symmetries, and the current phase relations for the charge supercurrent. Finally, concluding remarks are given in Sec. IV.

\section{METHODS}

To model a superconducting WS system, supporting type-I and type-II phases ${ }^{27,33}$, we use the following Bogoliubov-de Gennes Hamiltonian in spin-Nambu space:

$$
\mathcal{H}(\boldsymbol{k})=\left(\begin{array}{cc}
H(\boldsymbol{k})-\mu \hat{1} & \hat{\Delta} \\
\hat{\Delta}^{\dagger} & -H^{\mathrm{T}}(-\boldsymbol{k})+\mu \hat{1}
\end{array}\right),
$$

in which $\mu$ is the chemical potential, $k$ is the momentum of quasiparticles, $\hat{\Delta}$ is the superconducting gap, and $\mathrm{T}$ represents the transpose. The effective Hamiltonian $H_{\text {eff }}$ in the lowenergy regime, can be then expressed as,

$$
H_{\text {eff }}=\int \frac{d \boldsymbol{k}}{(2 \pi)^{3}} \check{\psi}_{\boldsymbol{k}}^{\dagger} \mathcal{H}(\boldsymbol{k}) \check{\psi}_{\boldsymbol{k}},
$$

where the associated $1 \times 8$ field operator for this Hamiltonian is given by $\breve{\psi}_{\boldsymbol{k}}^{\dagger}=\left[\hat{\psi}_{\boldsymbol{k}}^{\dagger}, \hat{\psi}_{-\boldsymbol{k}}\right]$, and $\hat{\psi}_{\boldsymbol{k}}^{\dagger}=\left(\psi_{A \uparrow}^{\dagger}, \psi_{A \downarrow}^{\dagger}, \psi_{B \uparrow}^{\dagger}, \psi_{B \downarrow}^{\dagger}\right)$, in which the Pspin (the band degree of freedom) and spin indices are denoted by $A, B$ and $\uparrow, \downarrow$, respectively.

The diagonal block elements of Eq. (1) are explicitly expressed as:

$$
\begin{gathered}
H(\boldsymbol{k})=\left(\beta+\gamma \tau_{z}\right)\left(k_{z}^{2}-Q^{2}\right)+\eta\left(k_{x}^{2}+k_{y}^{2}\right) \tau_{z}+ \\
\alpha_{x} k_{x} \tau_{x}+\alpha_{y} k_{y} \tau_{y}+\alpha_{z} k_{z} \tau_{z},
\end{gathered}
$$

where the two Weyl points are separated by $Q$ in momentum space. We will consider a broad range of orbital parameters $\alpha_{x, y, z}$, and tilt of the Weyl cones, i.e., $\beta$, which is responsible for driving the system into the type-II phase. We will also consider both positive and negative values of the bandstructure parameters $\beta$ and $\gamma$, with the latter being responsible for breaking time reversal symmetry and band splitting. Also, a nonzero $\alpha_{z}$ shifts the Weyl nodes in energies while $\alpha_{x, y}$ can induce a gap at the nodes. The dimension of $\beta, \gamma, \eta$ is $\mathrm{eV} \cdot \AA^{2}$ and $\alpha_{x, y, z}$ have units of $\mathrm{eV} \cdot \AA$. Also, the chemical potential that enters the formalism later has units of $\mathrm{eV}$ and the momenta are scaled using $\AA .{ }^{29-32}$ 
In what follows, we consider a situation where the superconductivity is a consequence of (1) opposite-Pspin and (2) equal-Pspin phonon-mediated mechanism. We also consider a model of opposite-spin electron-electron interactions throughout the paper. The first type of pairing mechanism is characterized by the following two-electron amplitudes:

$$
\Delta_{\uparrow \downarrow}^{A B}\left\langle\psi_{A \uparrow}^{\dagger} \psi_{B \downarrow}^{\dagger}\right\rangle+\text { H.c. }
$$

where $\Delta_{\uparrow \downarrow}^{A B}$ is the gap representing BCS spin-singlet phonon mediated electron-electron coupling between the $A$ and $B$ Pspins. The second type of pairing mechanism can be expressed by

$$
\begin{aligned}
& \Delta_{\uparrow \downarrow}^{A A}\left\langle\psi_{A \uparrow}^{\dagger} \psi_{A \downarrow}^{\dagger}\right\rangle+\text { H.c. }, \\
& \Delta_{\uparrow \downarrow}^{B B}\left\langle\psi_{B \uparrow}^{\dagger} \psi_{B \downarrow}^{\dagger}\right\rangle+\text { H.c. },
\end{aligned}
$$

where $\Delta_{\uparrow \downarrow}^{A A}$ and $\Delta_{\uparrow \downarrow}^{B B}$ correspond to the gap for BCS spin-singlet phonon mediated electron-electron coupling for each Pspin. In the following, to simplify notation, we suppress the indices so that when applicable, $\Delta_{\uparrow \downarrow}^{A B} \equiv \Delta$ and $\Delta_{\uparrow \downarrow}^{A A}=\Delta_{\uparrow \downarrow}^{B B} \equiv \Delta$.

We next consider the Green's functions for the Weyl semimetal system in the presence of superconductivity. The normal Green's functions $g$ and the anomalous Green's functions $f$ are defined as follows:

$$
\begin{aligned}
& g_{\rho \rho^{\prime}}^{\sigma \sigma^{\prime}}\left(\tau, \tau^{\prime} ; \mathbf{r}, \mathbf{r}^{\prime}\right)=-\left\langle\mathcal{T}_{\tau} \psi_{\sigma \rho}(\tau, \mathbf{r}) \psi_{\sigma^{\prime} \rho^{\prime}}^{\dagger}\left(\tau^{\prime}, \mathbf{r}^{\prime}\right)\right\rangle, \\
& \underline{g}_{\rho \rho^{\prime}}^{\sigma \sigma^{\prime}}\left(\tau, \tau^{\prime} ; \mathbf{r}, \mathbf{r}^{\prime}\right)=-\left\langle\mathcal{T}_{\tau} \psi_{\sigma \rho}^{\dagger}(\tau, \mathbf{r}) \psi_{\sigma^{\prime} \rho^{\prime}}\left(\tau^{\prime}, \mathbf{r}^{\prime}\right)\right\rangle, \\
& f_{\rho \rho^{\prime}}^{\sigma \sigma^{\prime}}\left(\tau, \tau^{\prime} ; \mathbf{r}, \mathbf{r}^{\prime}\right)=+\left\langle\mathcal{T}_{\tau} \psi_{\sigma \rho}(\tau, \mathbf{r}) \psi_{\sigma^{\prime} \rho^{\prime}}\left(\tau^{\prime}, \mathbf{r}^{\prime}\right)\right\rangle, \\
& f_{\rho \rho^{\prime}}^{\sigma \sigma^{\prime} \dagger}\left(\tau, \tau^{\prime} ; \mathbf{r}, \mathbf{r}^{\prime}\right)=+\left\langle\mathcal{T}_{\tau} \psi_{\sigma \rho}^{\dagger}(\tau, \mathbf{r}) \psi_{\sigma^{\prime} \rho^{\prime}}^{\dagger}\left(\tau^{\prime}, \mathbf{r}^{\prime}\right)\right\rangle,
\end{aligned}
$$

where $\mathcal{T}_{\tau}$ is the time ordering operator, and $\tau, \tau^{\prime}$ are the imaginary times. Here, $\rho, \rho^{\prime}$ and $\sigma, \sigma^{\prime}$ denote the spin and Pspin indices and $\langle\ldots\rangle$ denotes thermodynamic averaging. In particlehole space, the Green's function satisfies,

$$
\left(\begin{array}{cc}
\hat{H}(\mathbf{r})-i \omega_{n} & \hat{\Delta}(\mathbf{r}) \\
\hat{\Delta}^{*}(\mathbf{r}) & \mathcal{T}_{\mathrm{t}} \hat{H}(\mathbf{r}) \mathcal{T}_{\mathrm{t}}^{\dagger}+i \omega_{n}
\end{array}\right) \check{g}\left(i \omega_{n} ; \mathbf{r}, \mathbf{r}^{\prime}\right)=\delta\left(\mathbf{r}-\mathbf{r}^{\prime}\right),
$$

in which $\omega_{n}=\pi(2 n+1) k_{B} T$ is the Matsubara frequency, $n \in$ $Z, k_{B}$ is the Boltzman constant, $T$ is temperature, $\mathcal{T}_{\mathrm{t}}$ is the time reversal operator, and $\hat{\Delta}(\mathbf{r})$ is the superconducting gap in real space. Note that the Hamiltonian in real space $\hat{H}(\mathbf{r})$ is obtained by replacing $i k \equiv\left(\partial_{x}, \partial_{y}, \partial_{z}\right)$ in $H(\boldsymbol{k})$, in Eq. (3). The matrix form of the Green's function reads:

$$
\check{g}\left(i \omega_{n} ; \mathbf{r}, \mathbf{r}^{\prime}\right)=\left(\begin{array}{cc}
\hat{g}\left(i \omega_{n} ; \mathbf{r}, \mathbf{r}^{\prime}\right) & \hat{f}\left(i \omega_{n} ; \mathbf{r}, \mathbf{r}^{\prime}\right) \\
\hat{f}^{\dagger}\left(i \omega_{n} ; \mathbf{r}, \mathbf{r}^{\prime}\right) & \underline{\hat{g}}\left(i \omega_{n} ; \mathbf{r}, \mathbf{r}^{\prime}\right)
\end{array}\right),
$$

where we denote the $4 \times 4$ matrices by the symbol, $\hat{\ldots}$, and $8 \times 8$ matrices by the symbol, $\therefore$.

To calculate the supercurrent flowing through a Weyl semimetal Josephson junction, we assume a steady state situation where the charge density $\rho$ in the system is constant in time, namely, $\partial \rho / \partial t \equiv 0$. To ultimately calculate the charge current, we employ the quantum mechanical definition of the time-evolution of $\rho$ for a generic system that ensures charge conservation in the steady state, namely,

$$
\begin{array}{r}
\frac{\partial \rho}{\partial t}=\lim _{\boldsymbol{r} \rightarrow \boldsymbol{r}^{\prime}} \sum_{\sigma \rho v \sigma^{\prime} \rho^{\prime} v^{\prime}} \frac{1}{i \hbar}\left[\psi_{\sigma \rho v}^{\dagger}\left(\boldsymbol{r}^{\prime}\right) \mathcal{H}_{\sigma \rho v \sigma^{\prime} \rho^{\prime} v^{\prime}}(\boldsymbol{r}) \psi_{\sigma^{\prime} \rho^{\prime} v^{\prime}}(\boldsymbol{r})\right. \\
\left.-\psi_{\sigma \rho v}^{\dagger}\left(\boldsymbol{r}^{\prime}\right) \mathcal{H}_{\sigma \rho v \sigma^{\prime} \rho^{\prime} v^{\prime}}^{\dagger}\left(\boldsymbol{r}^{\prime}\right) \psi_{\sigma^{\prime} \rho^{\prime} v^{\prime}}(\boldsymbol{r})\right] .
\end{array}
$$

Here $\mathcal{H}_{\sigma \rho v \sigma^{\prime} \rho^{\prime} v^{\prime}}$ is the component form of Eq. (1) with the Pspin, spin, and particle-hole $\left(v, v^{\prime}\right)$ indices written explicitly. We now use the law of current conservation to arrive at the following quantum mechanical expression for the current density:

$$
\boldsymbol{J}=\int \boldsymbol{d} \boldsymbol{r}\left\{\hat{\psi}^{\dagger}(\boldsymbol{r}) \overrightarrow{\mathcal{H}}(\boldsymbol{r}) \hat{\psi}(\boldsymbol{r})-\hat{\psi}^{\dagger}(\boldsymbol{r}) \overleftarrow{\mathcal{H}}(\boldsymbol{r}) \hat{\psi}(\boldsymbol{r})\right\}
$$

where $\mathcal{H}(\boldsymbol{r})$ is given by Eq. (1), after the substitution $i \boldsymbol{k} \equiv$ $\left(\partial_{x}, \partial_{y}, \partial_{z}\right)$. The arrows point to the specific wavefunctions that are acted on by the derivative operators in the Hamiltonian. Note that any property of the system (including anisotropy and inhomogeneity) are indeed imbedded within the Hamiltonian of the system, associated wavefunctions, and boundary conditions (see below for more details).

\section{RESULTS AND DISCUSSIONS}

In this section we present results that depict representative behavior of the pair correlation profiles that may evolve in Weyl semimetal systems. We examine the momentum-space profiles and show that by varying experimentally relevant parameters, the effective symmetry classes of the superconducting correlations can undergo significant transformations. To compliment this, the charge supercurrent flow is then studied in a ballistic SNS Josephson structure, where a phase difference between the two superconducting electrodes is established. The direction and periodicity of supercurrent flow, and the possible emergence of $0-\pi$ transitions are investigated as a function of orbital effects, as well as tilt and spacing of the Weyl cones.

\section{A. Symmetry profile of superconducting pair correlations}

Here we present the anomalous Green's function $\hat{f}(i \omega ; \boldsymbol{k})$ and explore different symmetries that may arise by manipulating the Weyl semimetal material parameters. In the calculations that follow, we consider a representative node separation corresponding to $Q=0.4 \pi$, and note that our conclusions are independent of this specific choice. For simplicity, we consider the zeroth mode of the Matsubara frequency, following the fact that parity is not affected within the frequency domain. Each component of $\hat{f}(i \omega ; \boldsymbol{k})$ describes a specific pairing correlation function. To further simplify our calculations, we consider a system that is invariant in all directions so the momenta $k_{x, y, z}$ are good quantum numbers and can be treated algebraically in momentum space. By solving Eq. (7) in conjunction with the opposite-Pspin phonon mediated coupling 


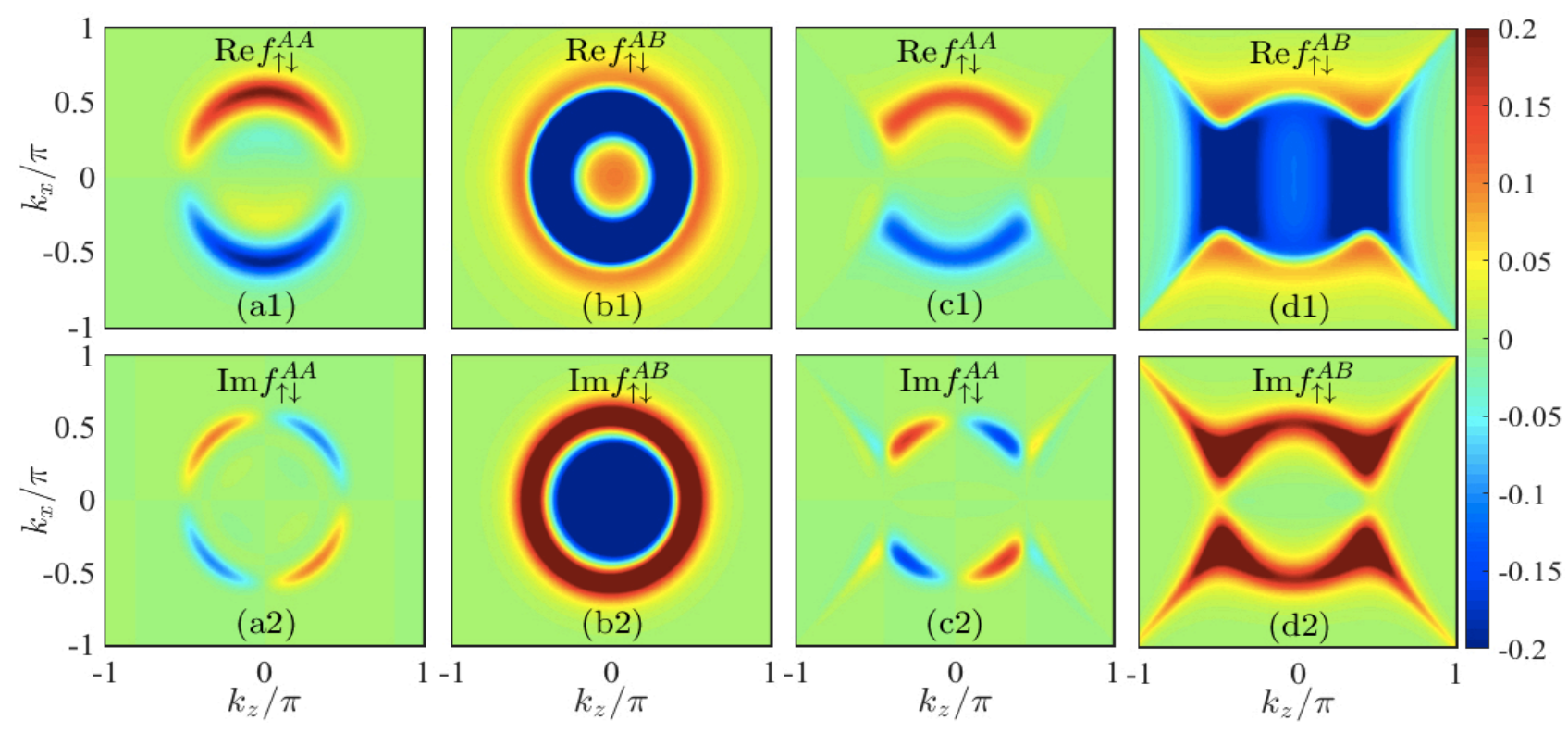

FIG. 1. (Color online). Real and imaginary parts of the superconducting correlations for the opposite-pseudospin phonon mediated electronelectron interaction plotted as a function of momenta $k_{x}$ and $k_{z}$. For illustrative purposes, the momentum component $k_{y}$ is set to zero. The following pair correlations are shown: $f_{\uparrow \downarrow}^{A A}$ and $f_{\uparrow \downarrow}^{A B}$. (a)-(b) $(\gamma>\beta)$ We use the representative parameters $\gamma=1.3, \beta=0.1, \alpha_{z}=\alpha_{x y}=0.2$, $\mu=0.5, \eta=1$, and $Q=0.4 \pi$. (c)-(d) $(\gamma<\beta)$ We use the representative parameters $\gamma=0.1, \beta=1.3, \alpha_{z}=\alpha_{x y}=0.2, \mu=0.5, \eta=1$, and $Q=0.4 \pi$

of Eq. (4), we end up with the following expressions for the superconducting correlations:

$$
\begin{aligned}
f_{\uparrow \uparrow}^{A A}= & 0 \\
f_{\uparrow \downarrow}^{A A}= & \frac{2}{\mathcal{D}} \alpha_{x y} \Delta \underbrace{\left(k_{x}-i k_{y}\right)}_{\mathcal{P}_{-}} \underbrace{(\Lambda+\mu+\Omega(\gamma-\beta))}_{\mathcal{D}_{-}}, \quad(11 \mathrm{a}) \\
f_{\uparrow \uparrow}^{A B}= & 0 \\
f_{\uparrow \downarrow}^{A B}= & -\frac{\Delta}{\mathcal{D}}[-(\Omega(11 \mathrm{~b}) \\
& \left(\Omega(\beta+\gamma)+\Lambda+i \omega_{n}\right)+\Delta^{2}+ \\
& \left.\alpha_{x y}^{2}\left(k_{x}^{2}+k_{y}^{2}\right)+\alpha_{z}^{2} k_{z}^{2}+2 \alpha_{z} k_{z}(\mu-\beta \Omega)-2 \mu \beta \Omega\right] \\
\mathcal{D}= & \left(k_{x}^{2}+k_{y}^{2}\right) \alpha_{x y}^{2}\left(-\mu^{2}+\left(k_{x}^{2}+k_{y}^{2}\right) \alpha_{x y}^{2}+\Delta^{2}+2 \mu\left(\beta \Omega-i \omega_{n}\right)+\left(k_{z} \alpha_{z}+\Lambda+(\beta+\gamma) \Omega-i \omega_{n}\right)\left(k_{z} \alpha_{z}+\Lambda-\beta \Omega+\gamma \Omega+i \omega_{n}\right)\right)+ \\
& \Delta^{2}\left(\left(k_{x}^{2}+k_{y}^{2}\right) \alpha_{x y}^{2}+\Delta^{2}-\left(\mu+k_{z} \alpha_{z}+\Lambda-\beta \Omega+\gamma \Omega+i \omega_{n}\right)\left(-\mu-k_{z} \alpha_{z}+\Lambda+(\beta+\gamma) \Omega+i \omega_{n}\right)\right)+ \\
& \left(\Delta^{2}\left(-\mu+k_{z} \alpha_{z}+\Lambda+(\beta+\gamma) \Omega-i \omega_{n}\right)+\left(\mu^{2}-\left(k_{x}^{2}+k_{y}^{2}\right) \alpha_{x y}^{2}-\left(k_{z} \alpha_{z}+\Lambda+(\beta+\gamma) \Omega-i \omega_{n}\right)\left(k_{z} \alpha_{z}+\Lambda-\beta \Omega+\gamma \Omega+i \omega_{n}\right)+\right.\right. \\
& \left.\left.\mu\left(-2 \beta \Omega+2 i \omega_{n}\right)\right)\left(-\mu-k_{z} \alpha_{z}+\Lambda+(\beta+\gamma) \Omega+i \omega_{n}\right)\right)\left(-\mu+k_{z} \alpha_{z}-\Lambda+\beta \Omega-\gamma \Omega+i \omega_{n}\right)
\end{aligned}
$$

where we have defined $\Omega=k_{z}^{2}-Q^{2}$, and $\Lambda=\eta\left(k_{x}^{2}+k_{y}^{2}\right)$. In a similar fashion, and using the symmetry relations appropriate for the Green's function components, one can obtain the other components of $\hat{f}(i \omega ; \boldsymbol{k})$. We have derived all components of $\check{g}(i \omega ; \boldsymbol{k})$, however, for the purposes shown in this paper, the components presented above are adequate.

Here, we have set $\alpha_{x}=\alpha_{y}=\alpha_{x y}$ to further simplify our

expressions. Note that if we set $\alpha_{x y}=0$, Eq. (12) reduces to,

$$
\begin{gathered}
\mathcal{D}=-\Delta^{2}+\left(-\mu+k_{z} \alpha_{z}-\Lambda+\beta \Omega-\gamma \Omega+i \omega_{n}\right) \\
\left(\mu-k_{z} \alpha_{z}-\Lambda-(\beta+\gamma) \Omega+i \omega_{n}\right),
\end{gathered}
$$



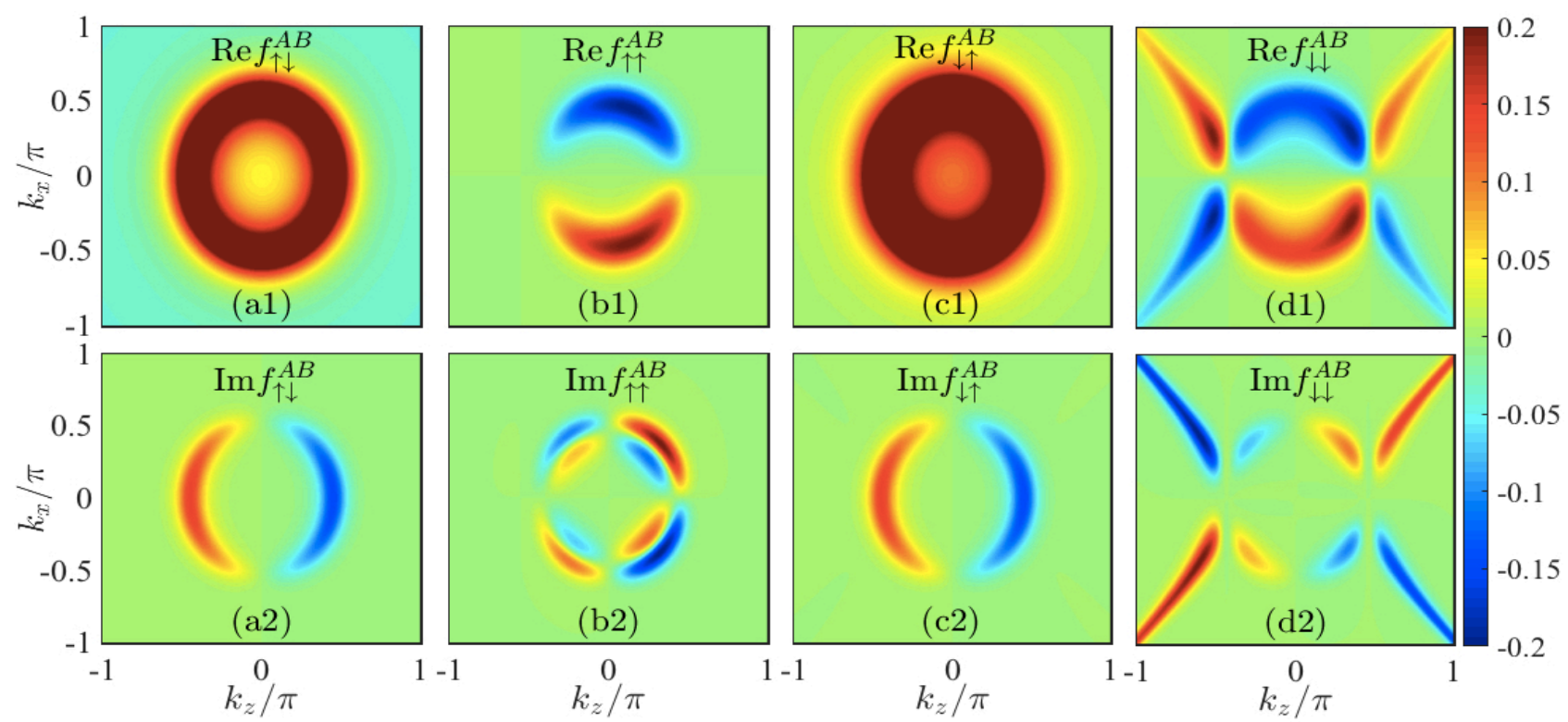

FIG. 2. (Color online). Real and imaginary parts of the superconducting correlations for the equal-pseudospin phonon mediated electronelectron interaction plotted as a function of momenta $k_{x}$ and $k_{z}$. The following pair correlations are shown: $f_{\uparrow \downarrow}^{A A}$ and $f_{\uparrow \downarrow}^{A B}$. The parameters here are the same as in Fig. 1.

and we also find,

$$
\begin{aligned}
& f_{\uparrow \uparrow}^{A A}=0, \\
& f_{\uparrow \downarrow}^{A A}=0, \\
& f_{\uparrow \uparrow}^{A B}=0, \\
& f_{\uparrow \downarrow}^{A B}=\frac{\Delta}{\mathcal{D}} .
\end{aligned}
$$

As seen, the orbital term is indeed responsible for inducing equal-Pspin pair correlations in WS so $f_{\uparrow \downarrow}^{A A}$ is directly proportional to $\alpha_{x, y}$. The expression governing the equal-Pspin component of the anomalous Green's function (when $\alpha_{x, y} \neq 0$ ) in Eq. (11b) has several important features: The numerator is proportional to $\mathcal{P}_{-}=k_{x}-i k_{y}$, which is of odd-parity, and corresponds to an effective $p$-wave symmetry $\left(p_{x}-i p_{y}\right)$. The second term in parentheses, $\mathcal{D}_{-}$, containing $\Omega$ and $\Lambda$, is of even-parity and has a symmetry expressible in the form $|a|\left(k_{x}^{2}+k_{y}^{2}\right) \pm|b| k_{z}^{2}$, where $a$ and $b$ are coefficients that depend on $\eta, \gamma$, and $\beta$. Specifically, from $\mathcal{D}_{+}=\Gamma+\mu+\Omega(\gamma+\beta)$, we find that $a \equiv \eta$ and $b \equiv(\gamma-\beta)$. Hence, depending on the choice of parameters (for example, in the regime where $\beta>\gamma$ or $\beta<\gamma$ ), the symmetry classification for the second term can be of the $s$-wave $\left(|a|\left(k_{x}^{2}+k_{y}^{2}\right)+|b| k_{z}^{2}\right)$ or $d$-wave $\left(|a|\left(k_{x}^{2}+k_{y}^{2}\right)-|b| k_{z}^{2}\right)$ types. Depending on the symmetry of $\mathcal{D}_{-}$, the numerator of Eq. (11b) belongs to the $p$-wave or $f$ wave symmetry classes. The complete symmetry profiles of $f_{\downarrow \uparrow}^{A A, B B}$ also depend on the symmetry of the denominator $\mathcal{D}$. Therefore, in what follows, we study effective symmetries of the superconducting pair correlations. Upon variations of the parameters $\eta, \gamma$, and $\beta$ in Eqs. (11b) and (11d), it is evident that the cumulative effect on $f_{\uparrow \downarrow}^{A A}$ and $f_{\uparrow \downarrow}^{A B}$ is to produce a spatial symmetry that can be classified as effectively $p$-wave and $f$-wave. Although $\mathcal{D}$ can have an effective $s$-wave symme- try, depending on $\eta, \gamma, \beta$, if $\alpha_{z}$ is nonzero, the overall symmetry of $f_{\uparrow \downarrow}^{A A}$ and $f_{\uparrow \downarrow}^{A B}$ can change as seen in Eqs. (12) and (13). Although more lengthy, an analysis of the components $f_{\uparrow \downarrow}^{B B}$, and $f_{\uparrow \downarrow}^{B A}$ reveals similar possibilities. For instance, if we set $\alpha_{z}=0$, we see that the numerators of $f_{\uparrow \downarrow}^{B A}$ can show either $s$-wave or $d$-wave effective symmetries, depending again, on the parameters $\eta, \gamma$, and $\beta$. Thus, for the phonon mediated unequal-Pspin scenario, the superconducting pairing correlations can change their effective spatial symmetry profiles from one symmetry class to another, simply by manipulating the material parameters of a Weyl semimetal. As recently demonstrated, control of these parameters is possible by, for example, applying strain to the system. ${ }^{29-32}$ Also, unconventional pairings and their symmetries were recently studied in systems with and without the lack of inversion symmetry. ${ }^{74-76}$ It is also worth noting that our results above illustrate that superconducting Weyl semimetals can develop odd-frequency symmetries. $28,31,32,72,81$

To further shed light on the symmetry profiles of these superconducting correlations, in Figs. 1-3, we present a few representative profiles for the real and imaginary parts of the equal-Pspin and opposite-Pspin components as a function of the momenta $k_{x}$ and $k_{y}$. The upper row of panels corresponds to the real parts, while the bottom row is for the imaginary parts. For simplicity, we consider zero momentum along $y$, $k_{y}=0$. We also consider here two regimes $(\beta<\gamma$ and $\beta>\gamma)$ and equal strengths of the orbital parameters: $\alpha_{z}=\alpha_{x y}=0.2$. In Figs. 1(a)-1(b) we implement a representative set of parameters: $\gamma=1.3, \beta=0.1, \mu=0.5, \eta=1$, and $Q=0.4 \pi$. As seen in Figs. 1(b1) and 1(b2), the effective symmetry of the $f_{\uparrow \downarrow}^{A B}$ correlations are of the $s$-wave type, which is indeed fully consistent with our discussion of Eq. (11d). Likewise, Figs. 1(a1) and 1(a2) show that the effective symmetry of both imaginary 


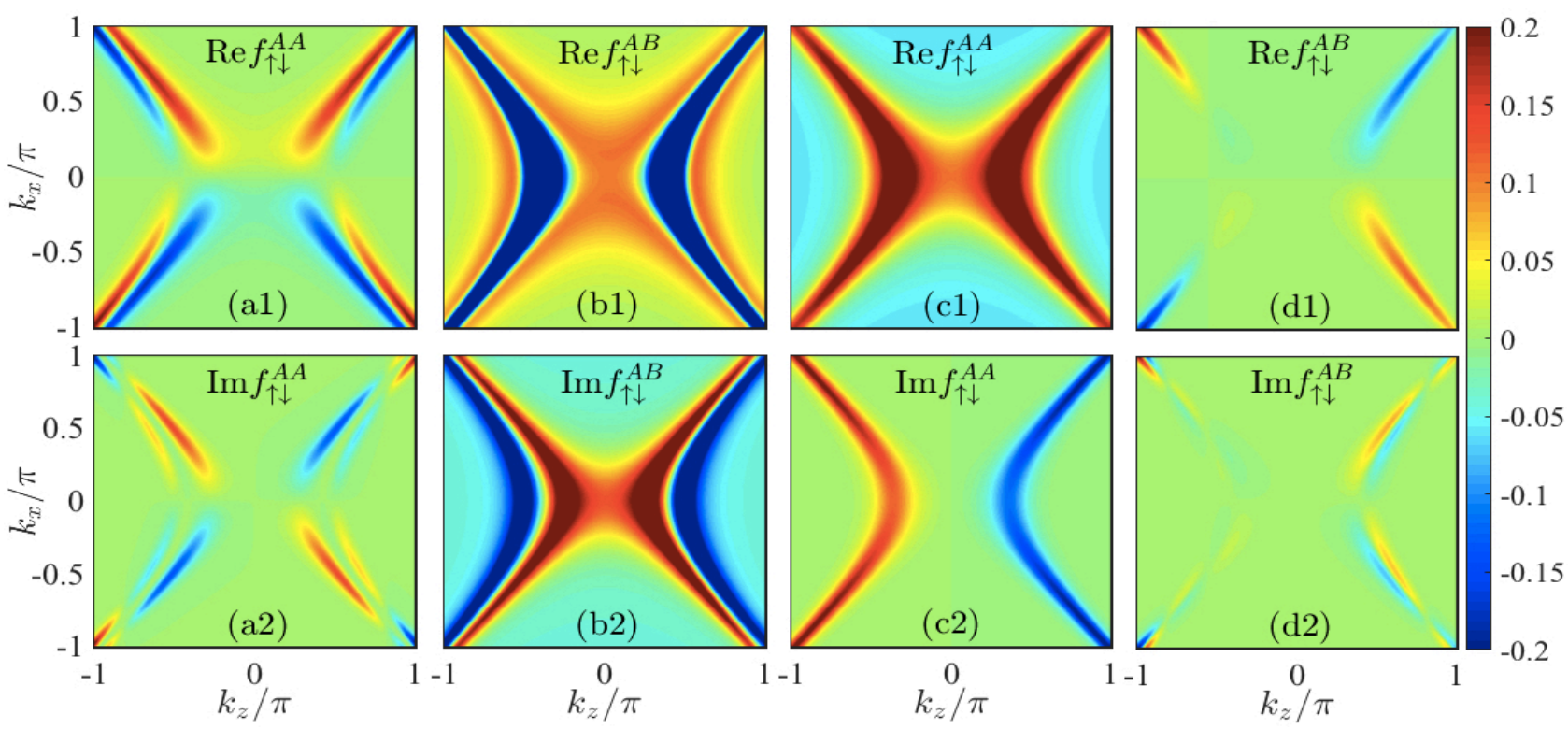

FIG. 3. (Color online). Real and imaginary parts of the superconducting correlations $f_{\uparrow \downarrow}^{A A}$ and $f_{\uparrow \downarrow}^{A B}$ plotted as a function of momenta $k_{x}$ and $k_{z}$. (a)-(b) The opposite-pseudospin phonon mediated electron-electron interaction is considered and parameters are identical to those of Figs. 1(a)-1(b) except we now set $\gamma=-1.3$. (c)-(d) The equal-pseudospin phonon mediated electron-electron interaction is set and parameters are identical to those of Figs. 2(a)-2(b), except now we flip the sign of $\gamma$ to minus $\gamma=-1.3$.

and real parts of $f_{\uparrow \downarrow}^{A A}$ are of the $p$-wave type, consistent with Eqs. (11b). We note that here we consider the regime where $\gamma>\beta$.

To illustrate how the effective $d$-wave and $f$-wave symmetries might evolve, we now set $\gamma=0.1$ and $\beta=1.3$, corresponding to the $\gamma<\beta$ regime. Note that, according to the discussion above, one is able to achieve similar symmetry changes in the effective symmetry profiles (described below) by tuning and/or reversing the sign of the parameters $\gamma, \eta$, and $\beta$. The resultant superconducting correlations are shown in Figs. 1(c)-1(d). We clearly observe in Figs. 1(b1), 1(b2), $1(\mathrm{~d} 1)$, and $1(\mathrm{~d} 2)$ that the previous effective $s$-wave symmetry has transformed into an effective $d$-wave class. Likewise, Figs. 1(a1), 1(a2), 1(c1), and 1(c2) illustrate that the previous effective $p$-wave symmetry has become of the $f$-wave type, which arises from the multiplication of terms with effective $p$-wave and $d$-wave symmetries, as discussed in connection with Eqs. (11b) and (11d). As for the effects of increasing the tilt parameter $\beta$, Eqs. (11) reveal that there are two competing effects that determine the final symmetry of the pair correlations: As $\beta$ increases, the pair correlations involving the term $(\gamma-\beta)$ decrease their $s$-wave character $\left[|a|\left(k_{x}^{2}+k_{y}^{2}\right)+|b| k_{z}^{2}\right]$, which is countered by the parameter $\gamma$ that drives the correlations into a $d$-wave symmetry class $\left[|a|\left(k_{x}^{2}+k_{y}^{2}\right)-|b| k_{z}^{2}\right]$. Thus, a multitude of possibilities arise for the pair correlation symmetries depending on the material properties and whether the Weyl semimetal is type-I or type-II.

To gain additional insight, we have also calculated the superconducting pairing correlations starting from an equalPspin scenario given by Eqs. (5). After performing the calculations, we find the following expressions for the components of anomalous Green's function:

$$
\begin{aligned}
f_{\uparrow \uparrow}^{A A}= & 0, \\
f_{\uparrow \downarrow}^{A A}= & \frac{1}{\mathcal{D}}\left[\Delta \left(\left(k_{x}-i k_{y}\right)^{2} \alpha_{x y}^{2}+\Delta^{2}+(\mu+\Lambda+\right.\right. \\
& \left.\left.(-\beta+\gamma) \Omega)^{2}-\left(k_{z} \alpha_{z}+i \omega_{n}\right)^{2}\right)\right], \\
f_{\uparrow \uparrow}^{A B}= & 0, \\
f_{\uparrow \downarrow}^{A B}= & -\frac{1}{\mathcal{D}}\left[2 \alpha_{x y} \Delta\left(k_{x}\left(\mu+k_{z} \alpha_{z}-\beta \Omega\right)+i k_{y}\left(\Lambda+\gamma \Omega+i \omega_{n}\right)\right)\right],
\end{aligned}
$$

$$
\begin{aligned}
\mathcal{D}= & -\left(-k_{x}+i k_{y}\right) \alpha_{x y}\left(\left(k_{x}-i k_{y}\right) \alpha_{x y} \Delta^{2}+\left(k_{x}+i k_{y}\right) \alpha_{x y}\left(-\mu^{2}+\left(k_{x}^{2}+k_{y}^{2}\right) \alpha_{x y}^{2}+2 \mu\left(\beta \Omega-i \omega_{n}\right)+\left(k_{z} \alpha_{z}+\Lambda+(\beta+\gamma) \Omega-i \omega_{n}\right)\right.\right. \\
& \left.\left.\left(k_{z} \alpha_{z}+\Lambda-\beta \Omega+\gamma \Omega+i \omega_{n}\right)\right)\right)+\Delta^{2}\left(\left(k_{x}+i k_{y}\right)^{2} \alpha_{x y}^{2}+\Delta^{2}+(-\mu+\Lambda+(\beta+\gamma) \Omega)^{2}-\left(k_{z} \alpha_{z}-i \omega_{n}\right)^{2}\right)+ \\
& \left(-\Delta^{2}\left(\mu+k_{z} \alpha_{z}+\Lambda-\beta \Omega+\gamma \Omega+i \omega_{n}\right)+\left(\mu^{2}-\left(k_{x}^{2}+k_{y}^{2}\right) \alpha_{x y}^{2}-\left(k_{z} \alpha_{z}+\Lambda+(\beta+\gamma) \Omega-i \omega_{n}\right)\right.\right. \\
& \left.\left.\left(k_{z} \alpha_{z}+\Lambda-\beta \Omega+\gamma \Omega+i \omega_{n}\right)+\mu\left(-2 \beta \Omega+2 i \omega_{n}\right)\right)\left(-\mu-k_{z} \alpha_{z}+\Lambda+(\beta+\gamma) \Omega+i \omega_{n}\right)\right)\left(-\mu+k_{z} \alpha_{z}-\Lambda+\beta \Omega-\gamma \Omega+i \omega_{n}\right) .
\end{aligned}
$$

We see that this kind of pairing interaction results in complicated expressions for the superconducting correlations,

specifically for the denominator. Therefore, we evaluate them numerically in Fig. 2. Similar to the previous case, if we set 
$\alpha_{x y}=0$, the expressions simplify considerably:

$$
\begin{aligned}
& f_{\uparrow \uparrow}^{A A}=0, \\
& f_{\uparrow \downarrow}^{A A}=\frac{\Delta}{\mathcal{D}}, \\
& f_{\uparrow \uparrow}^{A B}=0, \\
& f_{\uparrow \downarrow}^{A B}=0, \\
& \mathcal{D}=\Delta^{2}+(-\mu+\Lambda+(\beta+\gamma) \Omega)^{2}-\left(k_{z} \alpha_{z}-i \omega_{n}\right)^{2} .
\end{aligned}
$$

In agreement with our initial assumption for the electronelectron interactions [i.e., Eqs. (5)], we find that $f_{\uparrow \downarrow}^{A A}$ (or equivalently $f_{\uparrow \downarrow}^{B B}$ ) is the only nonzero component with even-parity when $\alpha_{z}=0$, which is evidently of the $s$-wave type. In Fig. 2, we have plotted the equal-Pspin and opposite-Pspin components of Green's function as a function of $k_{x}, k_{z}$, where $k_{y}=0$. The parameters set are identical to that used in Fig. 1. As seen in Figs. 2(a)-2(b) and 2(c)-2(d), when we move from the $\gamma>\beta$ regime to the regime where $\gamma<\beta$, only $f_{\uparrow \downarrow}^{A B}$ shows a discernible change in its spatial symmetry, while $f_{\uparrow \downarrow}^{A A}$ remains almost entirely intact. This is in contrast to the previous cases where both components $f_{\uparrow \downarrow}^{A B}$ and $f_{\uparrow \downarrow}^{A A}$ show changes in their symmetries. Nevertheless, we can conclude that the symmetry change is robust and can occur for the two electron-electron interaction scenarios considered in this paper (see also the following discussions).

Figure 3 illustrates the pairing correlations $f_{\uparrow \downarrow}^{A B}$ and $f_{\uparrow \downarrow}^{A A}$, where we have simply flipped the sign of $\gamma=-1.3$, and kept $\beta=0.1$ unchanged. In Figs. 3(a)-3(b), we plot the correlations for when the interaction between electrons with opposite spins are of the opposite-Pspins [Eq. (4)]. In contrast, Figs. 3(c)-3(d) correspond to equal-Pspin opposite-spin electron-electron interactions. It can be seen that by flipping the sign of $\gamma$, one can achieve pronounced symmetry changes in the superconducting correlations. The same feature can be observed when the sign of $\beta$ is reversed in the opposite regime where $\beta>\gamma$ (not shown).

Note that the possibility of symmetry change in spatial profiles of pair correlations relies on a sign change of the momenta coefficients. Such situations can be precisely determined by first-principles calculations; e.g., when the system experiences external strain or is subject to doping and intercalation. A practical example is when a black phosphorus monolayer is under the application of an external strain, as was recently discussed ${ }^{29-32}$. The band-structure parameters were obtained using density-functional-theory computations, and symmetry calculations demonstrated that the momenta coefficients can change signs depending on the strength and direction of the applied in-plane strain. Hence, angular pointcontact tunneling spectroscopy experiments or Meissner effect imaging are ideal probes to confirm the symmetry-change predictions discussed above.

An important quantity that can be additionally accounted for in our calculations and serve as a controlling parameter, is an exchange field ${ }^{29,30}$. Although we have not explicitly written it in our formulations, to incorporate an exchange field

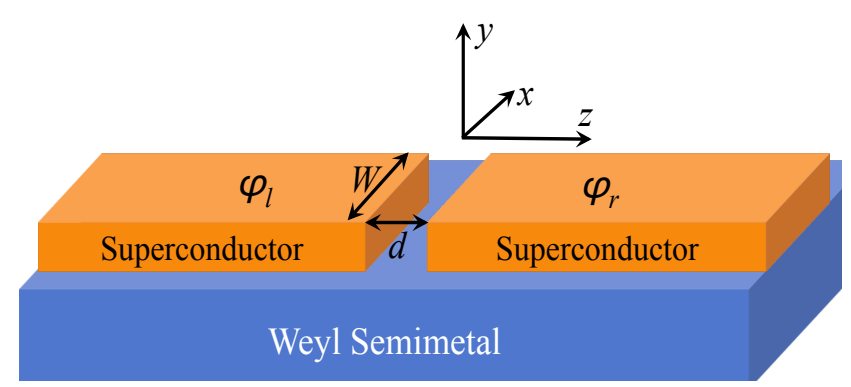

FIG. 4. (Color online). Schematic of a Weyl semimetal Josephson junction. The junction interfaces lie in the $x y$ plane, with the normal to the interfaces along the $z$ direction. The interfaces between the normal state Weyl semimetal and the superconductors are located at $z= \pm d / 2$. The width of the junction is $W$ and the macroscopic phase of the left and right superconductors are labeled by $\varphi_{l}$ and $\varphi_{r}$, respectively.

with three nonzero components $h_{i}$, the terms $h_{x} \rho_{x}+h_{y} \rho_{y}+h_{z} \rho_{z}$ should be attached to each Pspin [see Refs. 29-32], in which $\rho_{i}$ are Pauli matrices in spin space. We have investigated the superconducting pair correlations when the exchange field is nonzero in different directions. Our results (not shown) illustrate that the change of symmetry classes predicted above occurs for the spin-triplet superconducting correlations as well in the presence of an exchange field.

Our theoretical methods may also be extended to a scenario similar to the one investigated in a recent experiment that observed controlled superconductivity in $\mathrm{MoTe}_{2}$ by the application of an external mechanical strain ${ }^{66}$. It was observed that by the application of strain to $\mathrm{MoTe}_{2}$, the superconducting critical temperature $T_{c}$ increased. In consideration of this, it would be of interest to study $T_{c}$ for WS systems over a broad range of material and geometrical parameters. In this case, the superconducting gap function should be solved self-consistently to extract $T_{c}$, which is however, a nontrivial and formidable task that is postponed for future works. ${ }^{80,81}$

\section{B. Charge Supercurrent}

We now proceed to investigate the supercurrent flow through a Weyl Josephson junction. We consider a SNS type structure with a phase difference $\varphi=\varphi_{l}-\varphi_{r}$ established between the two superconducting electrodes [see Fig. 4]. To compute the total charge current, we take the current density perpendicular to the interface, which is in our case $j_{z}$, and integrate over the $x$ direction, i.e., $I(\varphi)=\int_{0}^{W_{x}} d x j_{z}(x, z, \varphi)$. Note that the current is independent of the $z$-coordinate as required by charge conservation. Upon diagonalizing $\mathcal{H}(\boldsymbol{r})$, we calculate the spinors $\psi_{\sigma \rho v}$ within each region. Next, To obtain the appropriate wavefunction in the normal region of the Josephson configuration, we match wavefunctions at the left $\hat{\psi}_{l}(z=-d / 2)=\hat{\psi}_{r}(z=-d / 2)$ and right boundaries $\hat{\psi}_{r}(z=+d / 2)=\hat{\psi}_{l}(z=+d / 2)$, and apply the continuity condition $\partial_{\boldsymbol{k}} \mathcal{H}_{l}(\boldsymbol{k}) \hat{\psi}_{l}=\partial_{\boldsymbol{k}} \mathcal{H}_{r}(\boldsymbol{k}) \hat{\psi}_{r}$, at the left and right superconductor interfaces. At the left boundary, $\mathcal{H}_{l}$ and $\mathcal{H}_{r}$ cor- 

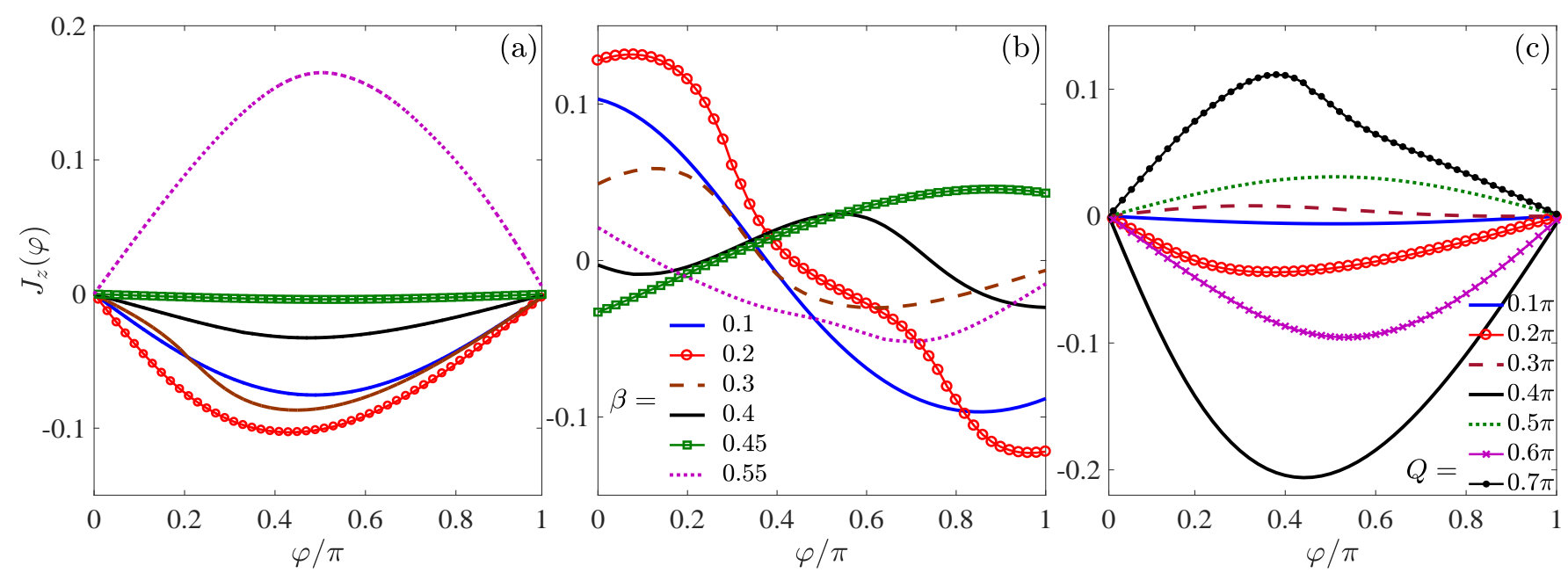

FIG. 5. (Color online). Normalized current flowing in the $z$ direction, $J_{z}(\varphi)$, as a function of the superconducting phase difference $\varphi$ applied across the junction for various values of the tilting parameter $\beta$ and separation of Weyl nodes $Q$ (see legends). In (a) the orbital term $\alpha_{z}$ is set zero. In (b) the orbital term $\alpha_{z}$ possesses a representative finite value of 0.2 . In (c) we set $\alpha_{z}$ zero and vary $Q$. The other parameters are set as follows: $\gamma=0.5, \beta=0.2, \alpha_{x, y}=1$, and $\eta=1$.

respond to the Hamiltonians of the superconducting and normal regions $\left(\hat{\psi}_{l}\right.$ and $\hat{\psi}_{r}$ are their associated wavefunctions), respectively, whereas at the right boundary they represent the Hamiltonians of the normal and superconducting regions, respectively. We note that, in order to accommodate a wide range of parameters without missing the important physics, we make no assumptions and approximations regarding the strength of the parameters involved that would normally simplify the wavefunctions. For example, the quasiclassical approximation assumes that $\mu$ is the largest energy in the system, which considerably simplifies the wavefunctions, but at the cost of losing important information for regimes where $\mu$ is small ${ }^{27-30}$. The resultant analytic expressions involving $1 \times 8$ spinors are therefore very cumbersome, and we omit the explicit expressions, presenting numerical results only when evaluating observable quantities. The junction length is fixed at a finite representative value $(d \approx 7.5 \mathrm{~nm})$, and we assume that the junction width $W$ is sufficiently large $(W \approx 100 \mathrm{~nm})$ so that boundary effects along the width direction are negligible. The central junction segment is undoped with $\mu_{N}=0$, while the chemical potential in the superconducting segments have the representative value $\mu_{S}=0.4$. It is further assumed that superconductivity is induced into the Weyl semimetal by virtue of proximity effects. From the component of the normalized current normal to the interfaces, $J_{z}$, the charge current flowing through the junction corresponds to $2 e J_{z}|\Delta| W / \hbar$. As seen from the components of the Hamiltonian in Eq. (3), the conical tilting parameter $\beta$ and the inversion symmetry breaking terms $\alpha_{x y}, \alpha_{z}$ are expected to strongly influence the flow of charge through the junction.

We show in Fig. 5, the normalized supercurrent $J_{z}$ as a function of $\varphi$ for a moderate range of the Weyl cone tilting parameter $\beta$ (see legend). Two different anisotropic states are considered: (a) where the orbital term vanishes $\alpha_{z}=0$, and (b) $\alpha_{z}=0.2$. When the orbital term is set to zero, we see in Fig. 5(a) that the supercurrent profile has the conventional sinusoidal $2 \pi$ periodicity. Increasing the tilt of the Weyl cones is shown to cause a non-monotonic variation in the amplitudes. Indeed, by tuning $\beta$ appropriately, the current can almost vanish or change direction altogether. Note that the current-phase periodicity changes to $4 \pi$ by the presence of higher harmonics at the current reversal points ${ }^{29,30}$. The appearance of higher order harmonics in the current-phaserelation can be made more pronounced by manipulating the WS parameter set (not shown). Next, in Fig. 5(b) the inversion symmetry breaking term takes a representative value corresponding to $\alpha_{z}=0.2$. By introducing a $z$-component to the inversion symmetry breaking term, the current-phaserelation undergoes a phase shift leading to a nonvanishing current at $\varphi=0, \pi$, creating a $\varphi_{0}$-state. Our results are consistent with recent findings in quasiclassical Weyl semimetal systems containing disorder ${ }^{28}$. Within a quasiclassical approach, it was found that supercurrent can flow through a triplet channel, and the $\varphi_{0}$ state is independent of the diffusion constant, demonstrating that this self-biased current can occur regardless of the density of nonmagnetic impurities and disorder in WS systems. Hence, the predicted robust self-biased current should be experimentally accessible over a wide range of parameters. ${ }^{28,29,77-79,82,83}$ It is worth mentioning that in a recent experiment, ${ }^{83}$ this self-biased current was observed using a $\mathrm{Bi}_{2} \mathrm{Se}_{3}$ platform, which is in agreement with theoretical predictions for topological insulator Josephson junctions ${ }^{77-79}$.

We also see that for type-II Weyl semimetals, increases in the conical tilt leads to a nontrivial current that is accompanied by the appearance of additional harmonics. In Fig. 5(c), we investigate the effects of varying the Weyl node separation parameter $Q$. Here we set $\alpha_{z}=0$, and $\beta=0.2$. We clearly see that this parameter can also control the direction and magnitude of the supercurrent, as well as the appearance of higher harmonics. In addition, the junction thickness $d$ can be ex- 


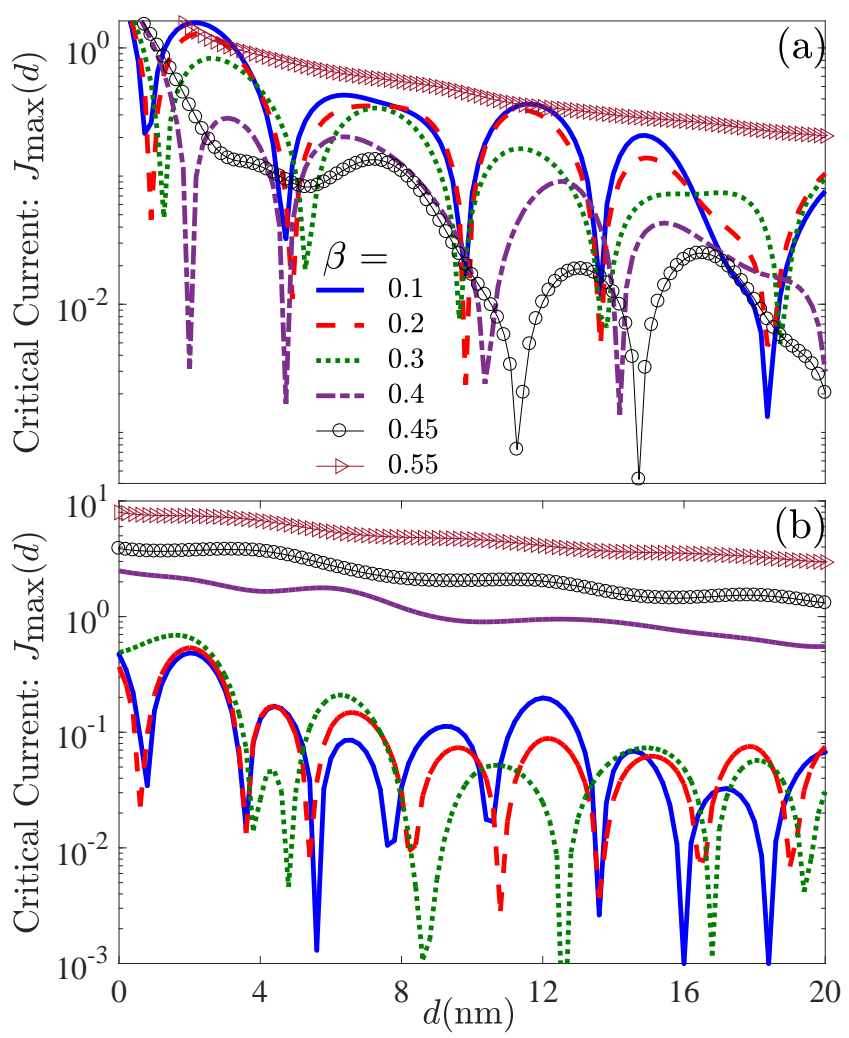

FIG. 6. (Color online). Critical normalized charge current $J_{\max }$ flowing across the Josephson junction shown in Fig. 5 as a function of junction thickness $d$. In (a) we set $Q=0.4 \pi$ and (b) $Q=0.7 \pi$ and show the influence of the tilting parameter $\beta$ on the critical supercurrent. The remaining junction parameters are set as follows: $\gamma=0.5$, $\alpha_{x, y}=1, \alpha_{z}=0$, and $\eta=1$.

ploited for controlling these phenomena in the supercurrent (not shown). Hence, our results demonstrate that a Josephson junction made of a type-I or type-II Weyl semimetal offers experimentally accessible parameters that can essentially act as control knobs to tune the supercurrent behavior. In many instances, these parameters are externally controllable, e.g., by applying an external strain ${ }^{29,30}$. For the exhibited sign reversals, manifestation of higher harmonics, and induction of a $\varphi_{0}$ state in the supercurrents shown above, no exchange interaction or Zeeman field was involved. This is in contrast to previously proposed platforms where the exchange field is an essential ingredient, and which might complicate experimental situations.

An experimentally relevant quantity is the critical supercurrent (maximum of the charge supercurrent defined by $\left.\max \left(\left|J_{z}(\varphi)\right|\right)\right)$ that can vary when one of the junction parameters changes. The generic behavior of the critical supercurrent in the Weyl semimetal Josephson junction we considered can be inferred from Fig. 5. For example, from Fig. 5(a) it is clear that the critical current in such a junction oscillates as a function of $\beta$. The behavior of the critical current can similarly be seen by considering other parameters, such as $Q$ (in Fig. 5(c)). These findings are summarized in the critical current plots shown in Fig. 6. To be consistent with the current phase relations presented in Fig. 5, we use identical parameters in Fig. 6. In Fig. 6(a) we set $Q=0.4 \pi$ and in Fig. 6(b) $Q=0.7 \pi$. The critical supercurrent is shown as a function of junction thickness $d$ for various values of the tilting parameter $\beta=0.1,0.2,0.3,0.4,0.45,0.55$. We see that the supercurrent undergoes multiple $0-\pi$ reversals by varying the junction thickness. Depending on $Q$ and the value for $\gamma$, increasing the tilting parameter beyond a threshold value causes the critical supercurrent to change its behavior drastically, as it reduces to a simple decaying function. This behavior originates from the now-dominate tilting parameter tilting parameter, which is consistent with Ref. ${ }^{28}$. Increasing $\beta$ results in amplifying the magnitude of the critical supercurrent. It is worth mentioning that we have purposefully chosen the orientation of the junction length to be along the $z$ direction. Because the Hamiltonian in Eq. (1) is not isotropic, the most interesting features of current transport in the model Weyl semimetal we consider appears when the current flows in the $z$ direction. This behavior follows from the interplay of broken timereversal and inversion symmetries in this specific direction. From Eq. (1), it is apparent that the interesting phenomena presented in Fig. 5 are absent when the Josephson junction is directed either along $x$ or $y$, so the SN and NS interfaces are oriented along $z$ ( $y$ or $x$ orientations, respectively). In particular, the appearance of the $\varphi_{0}$ self-biased Josephson state is specific to the charge current component flowing in the $z$ direction in our model Hamiltonian [Eq. (1)]. Lastly, it is worth mentioning that recent experiments involving $\mathrm{Cd}_{3} \mathrm{As}_{2}$ semimetal Josephson junctions have found $4 \pi$ periodic current phase relations ${ }^{84,85}$. This $4 \pi$ periodic current phase relation is attributed to the presence of topological superconductivity. Note that a $4 \pi$ periodic current phase relation can also appear in a ballistic Josephson junction with surface states of a three-dimensional topological insulator, and persist even in the quasiclassical regime where the chemical potential is large compared to other energy scales ${ }^{79}$.

\section{CONCLUSIONS}

Starting from equal-pseudospin and unequal-pseudospin phonon mediated spin-singlet electron pair couplings, we have studied the effective superconducting correlations that can exist in a Weyl semimetal system with the possible tilting of the Weyl cones, rendering the WS into type-II phase. We have considered a situation where the quasiparticle momenta are good quantum numbers, and derived the components of the Green's function that allow us to investigate the effective symmetry profiles of various superconducting correlations. Our results demonstrate that by properly engineering a Weyl semimetal system using intrinsic, and externally controllable parameters, the superconducting correlations can change symmetries from effective $s$-wave and $p$-wave classes to $d$-wave and $f$-wave types. Our results may provide a theoretical scenario for a recent experiment where it was observed that superconductivity in the $\mathrm{MoTe}_{2}$ Weyl semimetal depends on the applied strain of the system ${ }^{66}$. By diagonalizing the Bogoliubov-de Gennes Hamiltonian and deriving 
the corresponding wavefunctions ${ }^{29,30}$ (without any simplifying approximations ${ }^{28}$ ), we also found the supercurrent flow within a Josephson junction comprised of a ballistic Weyl semimetal. We demonstrated that a Weyl semimetal Josephson junction presents a practical platform to create a device that has several tunable parameters which control the direction and periodicity of supercurrent flow, induce multiple $0-\pi$ crossovers in the critical charge current, and generates a $\varphi_{0}$
Josephson state.

\section{ACKNOWLEDGMENTS}

M.A. is supported by Iran's National Elites Foundation (INEF). K.H. is supported in part by ONR and a grant of HPC resources from the DOD HPCMP.
${ }^{1}$ H.Z. Weyl, Elektron und Gravitation. I Physik 56, 330 (1929).

2 N.P. Armitage, E.J. Mele, and Ashvin Vishwanath, Weyl and Dirac semimetals in three-dimensional solids, Rev. Mod. Phys. 90, 015001 (2018)

${ }^{3}$ M. Z. Hasan and C. L. Kane, Colloquium: topological insulators, Rev. Mod. Phys. 82, 3045 (2010); X.-L. Qi and S.-C. Zhang, Topological insulators and superconductors, Rev. Mod. Phys. 83, 1057 (2011).

${ }^{4}$ S.-M. Huang, etal., A Weyl Fermion semimetal with surface Fermi arcs in the transition metal monopnictide TaAs class, Nat. Comm. 6, 7373 (2015).

5 B. Q. Lv, H. M. Weng, B. B. Fu, X. P. Wang, H. Miao, J. Ma, P. Richard, X. C. Huang, L. X. Zhao, G. F. Chen, Z. Fang, X. Dai, T. Qian, and H. Ding, Experimental discovery of Weyl semimetal TaAs, Phys. Rev. X 5, 031013 (2015).

${ }^{6}$ I. Belopolski, S. Y. Xu, D. S. Sanchez, G. Chang, C. Guo, M. Neupane, H. Zheng, C. C. Lee, S. M. Huang, G. Bian, N. Alidoust, T. R. Chang, B. K. Wang, X. Zhang, A. Bansil, H. T. Jeng, H. Lin, S. Jia, and M. Z. Hasan, Criteria for directly detecting topological Fermi arcs in Weyl semimetals, Phys. Rev. Lett. 116, 066802 (2016).

7 A. A. Burkov and L. Balents, Weyl semimetal in a topological insulator multilayer, Phys. Rev. Lett. 107, 127205 (2011).

8 A. A. Soluyanov, D. Gresch, Z. Wang, Q. Wu, M. Troyer, X. Dai, and B. A. Bernevig, Type-II Weyl semimetals, Nature (London) 527, 495 (2015).

9 S.-Y. Xu, N. Alidoust, G. Chang, H. Lu, B. Singh, I. Belopolski, D. Sanchez, X. Zhang, G. Bian, H. Zheng, M.-A. Husanu, Y. Bian, S.-M. Huang, C.-H. Hsu, T.-R. Chang, H.-T. Jeng, A. Bansil, V. N. Strocov, H. Lin, S. Jia, M. Z. Hasan, Discovery of Lorentz-violating Weyl fermion semimetal state in LaAlGe materials, arXiv:1603.07318.

${ }^{10}$ F. Detassis, L. Fritz, and S. Grubinskas, Collective effects in tilted Weyl cones: Optical conductivity, polarization, and Coulomb interactions reshaping the cone, Phys. Rev. B 96, 195157 (2017).

11 S. P. Mukherjee and J. P. Carbotte, Absorption of circular polarized light in tilted type-I and type-II Weyl semimetals, Phys. Rev. B 96, 085114 (2017).

12 S. P. Mukherjee and J. P. Carbotte, Imaginary part of Hall conductivity in a tilted doped Weyl semimetal with both broken timereversal and inversion symmetry, Phys. Rev. B 97, 035144 (2018).

13 K. Halterman, M. Alidoust, and A. Zyuzin, Epsilon-near-zero response and tunable perfect absorption in Weyl semimetals, Phys. Rev. B 98, 085109 (2018).

${ }^{14} \mathrm{~K}$. Halterman and M. Alidoust, Waveguide modes in Weyl semimetals with tilted Dirac cones, Opt. Express 27, 36182 (2019).

15 S. A. Yang, H. Pan, and F. Zhang, Dirac and Weyl superconductors in three dimensions, Phys. Rev. Lett. 113, 046401 (2014).
16 F. Setiawan, W. S. Cole, J. D. Sau, and S. Das Sarma, Transport in superconductornormal metalsuperconductor tunneling structures: Spinful p-wave and spin-orbit-coupled topological wires, Phys. Rev. B 95, 174515 (2017); F. Setiawan, A. Stern, E. Berg, Topological superconductivity in planar Josephson junctionsnarrowing down to the nanowire limit, arXiv:1902.11300.

17 A. Fornieri, A. M. Whiticar, F. Setiawan, E. P. Marn, A. C. C. Drachmann, A. Keselman, S. Gronin, C. Thomas, T. Wang, R. Kallaher, G. C. Gardner, E. Berg, M. J. Manfra, A. Stern, C. M. Marcus, F. Nichele, Evidence of topological superconductivity in planar Josephson junctions, arXiv:1809.03037.

18 A. Matos-Abiague, J. Shabani, A. D. Kent, G. L. Fatin, B. Scharf, I. uti, Tunable magnetic textures: From Majorana bound states to braiding, Solid State Commun. 262, 1 (2017).

19 T. Zhou, N. Mohanta, J. E. Han, A. Matos-Abiague, I. Zutic, From spintronics to Majorana bound states, arXiv:1901.02506.

20 Y. Qi, etal., Superconductivity in Weyl semimetal candidate $\mathrm{MoTe}_{2}$, Nature Comm. 7, 11038 (2016).

21 S.-Y. Xu, I. Belopolski, N. Alidoust, M. Neupane, G. Bian, C. Zhang, R. Sankar, G. Chang, Z. Yuan, C.-C. Lee, S.-M. Huang, H. Zheng, J. Ma, D. S. Sanchez, B. Wang, A. Bansil, F. Chou, P. P. Shibayev, H. Lin, S. Jia, and M. Z. Hasan, Discovery of a Weyl fermion semimetal and topological Fermi arcs Science 349, 613 (2015).

${ }^{22}$ M.D. Bachmann, N. Nair, F. Flicker, R. Ilan, T. Meng, N.J. Ghimire, E.D. Bauer, F. Ronning, J.G. Analytis, and P.J.W Moll, Inducing Superconductivity in Weyl Semimetal Microstructures by Selective Ion Sputtering, Sci. Adv. 3, e1602983 (2017).

23 P. Goswami, A. H. Nevidomskyy, Phys. Rev. B 92, 214504 (2015).

${ }^{24}$ G. Y. Cho, J. H. Bardarson, Y.-M. Lu, and J. E. Moore, Superconductivity of doped Weyl semimetals: Finite-momentum pairing and electronic analog of the ${ }^{3} \mathrm{He}-\mathrm{A}$ phase, Phys. Rev. B 86, 214514 (2012).

25 T. Zhou, Y. Gao, and Z. D. Wang, Superconductivity in Weyl metals, Phys. Rev. B 93, 094517 (2016).

${ }^{26}$ P. Hosur, X. Dai, Z. Fang, and X.-L. Qi, Time-reversal-invariant topological superconductivity in doped Weyl semimetals, Phys. Rev. B 90, 045130 (2014).

${ }^{27}$ M. Alidoust, K. Halterman, and A. A. Zyuzin, Superconductivity in type-II Weyl semimetals, Phys. Rev. B 95, 155124 (2017).

${ }^{28}$ M. Alidoust, Self-biased current, magnetic interference response, and superconducting vortices in tilted Weyl semimetals with disorder, Phys. Rev. B 98, 245418 (2018).

29 M. Alidoust, M. Willatzen, A.-P. Jauho, Strain-engineered Majorana zero energy modes and $\varphi_{0}$ Josephson state in black phosphorus, Phys. Rev. B 98, 085414 (2018).

${ }^{30}$ M. Alidoust, M. Willatzen, A.-P. Jauho, Fraunhofer response and supercurrent spin switching in black phosphorus with strain and disorder, Phys. Rev. B 98, 184505 (2018). 
31 M. Alidoust, M. Willatzen, A.-P. Jauho, Control of superconducting pairing symmetries in monolayer black phosphorus, Phys. Rev. B 99, 125417 (2019).

${ }^{32}$ M. Alidoust, M. Willatzen, A.-P. Jauho, Symmetry of superconducting correlations in displaced bilayers of graphene, Phys. Rev. B 99, 155413 (2019).

${ }^{33}$ G. Bednik, A. A. Zyuzin, and A. A. Burkov, Superconductivity in Weyl metals, Phys. Rev. B 92, 035153 (2015).

${ }^{34}$ R. C. Xiao, P. L. Gong, Q. S. Wu, W. J. Lu, M. J. Wei, J. Y. Li, H. Y. Lv, X. Luo, P. Tong, X. B. Zhu, and Y. P. Sun, Manipulation of type-I and type-II Dirac points in $\mathrm{PdTe}_{2}$ superconductor by external pressure, Phys. Rev. B 96, 075101 (2017).

35 B. Rosenstein, B.Y. Shapiro, D. Li, I. Shapiro, Magnetic properties of type-I and type-II Weyl semimetals in the superconducting state, Phys. Rev. B 97, 144510 (2018).

${ }^{36}$ Z. Hou and Q. Sun, Double Andreev Reflections in Type-II Weyl Semimetal-Superconductor Junctions, Phys. Rev. B 96, 155305 (2017).

37 X.-S. Li, S.-F. Zhang, X.-R. Sun, W.-J. Gong, Double Andreev reflections and double electron transmissions in a normalsuperconductor-normal junction based on type-II Weyl semimetal, arXiv: 1805.07928.

38 S.-B. Zhang, F. Dolcini, D. Breunig, B. Trauzettel, Appearance of the universal value $e^{2} / h$ of the zero-bias conductance in a Weyl semimetal-superconductor junction, Phys. Rev. B 97, 041116 (2018).

39 S.-B. Zhang, J. Erdmenger, B. Trauzettel, Chirality Josephson current due to a novel quantum anomaly in inversion-asymmetric Weyl semimetals, arXiv:1806.08111.

40 A. Chen, D. I. Pikulin, and M. Franz, Josephson current signatures of Majorana flat bands on the surface of time-reversalinvariant Weyl and Dirac semimetals, Phys. Rev. B 95, 174505 (2017).

41 Y. Xu, S. Uddin, J. Wang, Z. Ma, and J.-F. Liu, Electrically modulated SQUID with a single Josephson junction coupled by a time reversal breaking Weyl semimetal thin film, Phys. Rev. B 97, 035427 (2017).

42 J. Fang, W. Duan, J. Liu, C. Zhang, and Z. Ma, Pairing-dependent superconductivity gap and nonholonomic Andreev reflection in Weyl semimetal/Weyl superconductor heterojunctions, Phys. Rev. B 97, 165301 (2018).

${ }^{43}$ N. Bovenzi, M. Breitkreiz, P. Baireuther, T. E. OBrien, J. Tworzydlo, I. Adagideli, and C. W. J. Beenakker, Chirality blockade of Andreev reflection in a magnetic Weyl semimetal, Phys. Rev. B 96, 035437 (2017).

${ }^{44}$ S. Das, Amit, A. Sirohi, L. Yadav, S. Gayen, Y. Singh, and G. Sheet, Conventional Superconductivity in Type-II Dirac Semimetal PdTe2, Phys. Rev. B 97, 014523 (2017).

45 A. Kononov, O.O. Shvetsov, S.V. Egorov, A.V. Timonina, N.N. Kolesnikov, E.V. Deviatov, Signature of Fermi arc surface states in Andreev reflection at the $\mathrm{WTe}_{2}$ Weyl semimetal surface, EuroPhys. Lett. 122, 27004 (2018).

46 A. Kononov, O.O. Shvetsov, S.V. Egorov, A.V. Timonina, N.N. Kolesnikov, E.V. Deviatov, $\pi$ periodicity in Josephson effect for a three-dimensional Weyl semimetal $\mathrm{WTe}_{2}$, Euro. Phys. Lett. 124, 47003 (2018).

${ }^{47}$ S. Teknowijoyo, N. H. Jo, M. S. Scheurer, M. A. Tanatar, K. Cho, S. L. Bud'ko, P. P. Orth, P. C. Canfield, R. Prozorov, Nodeless superconductivity in type-II Dirac semimetal $\mathrm{PdTe}_{2}$ : lowtemperature London penetration depth and symmetry analysis, Phys. Rev. B 98, 024508 (2018).

48 A. Sirohi, S. Das, R. R. Chowdhuri, A., Y. Singh, S. Gayen, G. Sheet, Mixed type I and type-II superconductivity due to intrinsic electronic inhomogeneities in the type-II Dirac semimetal $\mathrm{PdTe}_{2}$,
arXiv: 1804.10837.

49 P. Lu, J.-S. Kim, J. Yang, H. Gao, J. Wu, D. Shao, B. Li, D. Zhou, J. Sun, D. Akinwande, D. Xing, and J.-F. Lin, Origin of superconductivity in the Weyl semimetal $\mathrm{WTe}_{2}$ under pressure, Phys. Rev. B 94, 224512 (2016).

${ }^{50}$ H. Takahashi, T. Akiba, K. Imura, T. Shiino, K. Deguchi, N. K. Sato, H. Sakai, M. S. Bahramy, and S. Ishiwata, Anticorrelation between polar lattice instability and superconductivity in the Weyl semimetal candidate $\mathrm{MoTe}_{2}$, Phys. Rev. B 95, 100501(R) (2017).

51 Z. Guguchia, F. von Rohr, Z. Shermadini, A. T. Lee, S. Banerjee, A. R. Wieteska, C. A. Marianetti, B. A. Frandsen, H. Luetkens, Z. Gong, S. C. Cheung, C. Baines, A. Shengelaya, G. Taniashvili, A. N. Pasupathy, E. Morenzoni, S. J. L. Billinge, A. Amato, R. J. Cava, R. Khasanov, and Y. J. Uemura, Signatures of the topological $s^{ \pm}$superconducting order parameter in the type-II Weyl semimetal $\mathrm{T}_{\mathrm{d}} \mathrm{MoTe}_{2}$, Nat. Comm. 8, 1082 (2017).

52 Q. Li, C. He, Y. Wang, E. Liu, M. Wang, Y. Wang, J. Zeng, Z. Ma, T. Cao, C. Yi, N. Wang, K. Watanabe, T. Taniguchi, L. Shao, Y. Shi, X. Chen, S.-J. Liang, Q.-H. Wang, F. Miao, ProximityInduced Superconductivity with Subgap Anomaly in Type-II Weyl Semi-Metal $\mathrm{WTe}_{2}$, Nano Lett. (2018).

53 Y. Xing, Z. Shao, J. Ge, J. Wang, Z. Zhu, J. Liu, Y. Wang, Z. Zhao, J. Yan, D. Mandrus, B. Yan, X.-J. Liu, M. Pan, J. Wang, Surface Superconductivity in the type II Weyl Semimetal TaIrTe ${ }_{4}$, arXiv:1805.10883.

${ }^{54}$ D. Li, B. Rosenstein, B. Ya. Shapiro, and I. Shapiro, Effect of the type I to type II Weyl semimetal topological transition on superconductivity, Phys. Rev. B 95, 094513 (2017).

$55 \mathrm{H}$. Wei, S. Chao, and V. Aji, Odd-parity superconductivity in Weyl semimetals, Phys. Rev. B 89, 014506 (2014).

56 B. Lu, K. Yada, M. Sato, and Y. Tanaka, Crossed surface flat bands of Weyl semimetal superconductors, Phys. Rev. Lett. 114, 096804 (2015).

57 R. Wang, L. Hao, B. Wang, C. S. Ting, Quantum anomalies in superconducting Weyl metals, Phys. Rev. B 93, 184511 (2016).

58 Y. Li, F. D. M. Haldane, Topological nodal Cooper pairing in doped Weyl metals, Phys. Rev. Lett. 120, 067003 (2018).

59 T. Meng and L. Balents, Weyl superconductors, Phys. Rev. B 86, 054504 (2012).

60 T. Das, Weyl semimetal and superconductor designed in an orbital-selective superlattice, Phys. Rev. B 88, 035444 (2013).

61 U. Khanna, A. Kundu, S. Pradhan, and S. Rao, Proximity-induced superconductivity in Weyl semimetals, Phys. Rev. B 90, 195430 (2014).

${ }^{62}$ E. V. Gorbar, V. A. Miransky, I. A. Shovkovy, P. O. Sukhachov, Inter-node superconductivity in strained Weyl semimetals, J. Phys.: Condens. Matter 31, 055602 (2019).

63 C. H. Wu, Electric Field and Light Field Modulated Josephson Effect in Silicene-Based SNS Josephson Junction: Andreev Reflection and Free Energy, Silicon (2019).

64 T. Zhou, Y. Gao, and Z. D. Wang, Resolving different pairing states in Weyl superconductors through the single-particle spectrum, Phys. Rev. B 98, 024515 (2018).

65 A. Vasdev, A. Sirohi, M.K. Hooda, C.S Yadav, G. Sheet, Enhanced, homogeneous type II superconductivity in $\mathrm{Cu}$ intercalated $\mathrm{PdTe}_{2}$, arXiv:1903.04321.

66 C. Heikes, I.-L. Liu, T. Metz, C. Eckberg, P. Neves, Y. Wu, L. Hung, P. Piccoli, H. Cao, J. Leao, J. Paglione, T. Yildirim, N. P. Butch, W. Ratcliff, Mechanical control of crystal symmetry and superconductivity in Weyl semimetal $\mathrm{MoTe}_{2}$, Phys. Rev. Materials 2, 074202 (2018).

${ }^{67}$ M. Alidoust, K. Halterman, and O. T. Valls, Zero Energy Peak and Triplet Correlations in Nanoscale SFF Spin-Valves, Phys. Rev. B 92, 014508 (2015). 
68 Y. Tanaka and A. A. Golubov, Theory of the Proximity Effect in Junctions with Unconventional Superconductors, Phys. Rev. Lett. 98, 037003 (2007).

${ }^{69}$ L. Kuerten, C. Richter, N. Mohanta, T. Kopp, A. Kampf, J. Mannhart, and H. Boschker, In-gap states in superconducting $\mathrm{LaAlO}_{3} \mathrm{SrTiO}_{3}$ interfaces observed by tunneling spectroscopy, Phys. Rev. B 96, 014513 (2017).

${ }^{70}$ G. Koren, T. Kirzner, Y. Kalcheim, and O. Millo, Signature of proximity-induced $\mathrm{p}_{\mathrm{x}}+\mathrm{ip}_{\mathrm{y}}$ triplet pairing in the doped topological insulator $\mathrm{Bi}_{2} \mathrm{Se}_{3}$ by the s-wave superconductor $\mathrm{NbN}$, EuroPhys. Lett. 103, 67010 (2013).

${ }^{71}$ G. Koren, Strongly suppressed proximity effect and ferromagnetism in topological insulator/ferromagnet/superconductor thin film trilayers of $\mathrm{Bi}_{2} \mathrm{Se}_{3} / \mathrm{SrRuO}_{3}$ underdoped $\mathrm{YBa}_{2} \mathrm{Cu}_{3} \mathrm{O}_{\mathrm{x}}$ : a possible new platform for Majorana nano-electronics, Supercond. Sci. Technol. 31, 075004 (2018).

72 M. Alidoust, A. Zyuzin, K. Halterman, Pure Odd-Frequency Superconductivity at the Cores of Proximity Vortices, Phys. Rev. B 95, 045115 (2017).

${ }^{73}$ M. Alidoust, K. Halterman, and J. Linder, Singlet-Triplet Superconducting Quantum Magnetometer, Phys. Rev. B 88, 075435 (2013).

74 Y. Fukaya, K. Yada, A. Hattori, Y. Tanaka, Pairing mechanism of unconventional superconductivity in doped Kane-Mele model, J. Phys. Soc. Jpn. 85, 104704 (2016).

75 Y. Fukaya, S. Tamura, K. Yada, Y. Tanaka, P. Gentile, M. Cuoco, Inter-orbital topological superconductivity in spin-orbit coupled superconductors with inversion symmetry breaking, Phys. Rev. B 97, 174522 (2018).

76 Y. Fukaya, S. Tamura, K. Yada, Y. Tanaka, P. Gentile, M. Cuoco, Spin-Orbital Hallmarks of Unconventional Superconductors Without Inversion Symmetry, arXiv:1903.12379.

77 A. Zyuzin, M. Alidoust, D. Loss, Josephson junction through a disordered topological insulator with helical magnetization, Phys.
Rev. B 93, 214502 (2016).

78 I. V. Bobkova, A. M. Bobkov, A. A. Zyuzin, M. Alidoust, Magnetoelectrics in disordered topological insulator Josephson junctions, Phys. Rev. B 94, 134506 (2016).

79 M. Alidoust and H. Hamzehpour, Spontaneous supercurrent and $\varphi_{0}$ phase shift parallel to magnetized topological insulator interfaces, Phys. Rev. B 96, 165422 (2017).

80 K. Halterman and M. Alidoust, Half-metallic superconducting triplet spin valve, Phys. Rev. B 94, 064503 (2016).

${ }^{81}$ M. Alidoust and K. Halterman, Half-Metallic Superconducting Triplet Spin MultiValves, Phys. Rev. B 97, 064517 (2018).

${ }^{82}$ V. Braude and Yu. V. Nazarov, Fully Developed Triplet Proximity Effect, Phys. Rev. Lett. 98, 077003 (2007); M. Eschrig and T. Lofwander, Triplet supercurrents in clean and disordered halfmetallic ferromagnets, Nat. Phys. 4, 138 (2008); A. Buzdin, Direct Coupling Between Magnetism and Superconducting Current in the Josephson Junction, ibid 101, 107005 (2008); Y. Tanaka, T. Yokoyama, and N. Nagaosa, Manipulation of the Majorana Fermion, Andreev Reflection, and Josephson Current on Topological Insulators, ibid. 103, 107002 (2009).

83 A. Assouline, C. Feuillet-Palma, N. Bergeal, T. Zhang, A. Mottaghizadeh, A. Zimmers, E. Lhuillier, M. Marangolo, M. Eddrief, P. Atkinson, M. Aprili, H. Aubin, Spin-Orbit induced phase-shift in $\mathrm{Bi}_{2} \mathrm{Se}_{3}$ Josephson junctions, Nat. Comm. 10, 126 (2019).

${ }^{84}$ W. Yu, W. Pan, D. L. Medlin, M. A. Rodriguez, S. R. Lee, Zhiqiang Bao, and F. Zhang, $\pi$ and $4 \pi$ Josephson Effects Mediated by a Dirac Semimetal, Phys. Rev. Lett. 120, 177704 (2018).

85 A.-Q. Wang, C.-Z. Li, C. Li, Z.-M. Liao, A. Brinkman, and D.-

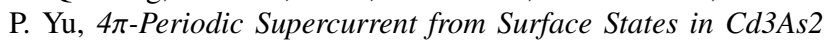
Nanowire-Based Josephson Junctions, Phys. Rev. Lett. 121, 237701 (2018). 